consultants who had a case load of more than 100 children with inflammatory bowel disease. Remaining $40 \%$ responses were made up by consultant paediatricians with gastroenterology interest and specialist nurses.

Less than half of the participants reported that they do routine screening for iron deficiency as part of IBD management. The lab parameters used to confirm iron deficiency varied significantly. In our survey, $28 \%$ of the participants were unsure or did not agree correcting iron deficiency anaemia alone will improve the quality of life in IBD patients if underlying disease control could not be improved. While majority of the participants (48.5\%) used oral iron as the first line treatment, some (8.5\%) were using IV iron as their first line. Our survey showed that only $11.4 \%$ of the participants reported that they had a local or regional guideline.

Conclusion Our survey highlights that there is significant variation in practise among the clinicians in methods of diagnosing and treating iron deficiency anaemia in children with IBD. It also underscores the need for evidence based national guidelines in this area.

\section{G202(P) A SURVEY OF NECROTISING ENTEROCOLITIS AT A TERTIARY NEONATAL UNIT}

doi:10.1136/archdischild-2013-304107.214

${ }^{1}$ A Wilcock, ${ }^{2} S$ Victor. 'Manchester Medical School, University of Manchester, Manchester, UK; ${ }^{2}$ Faculty of Medical and Human Sciences, University of Manchester, Manchester, UK

Aims Necrotising enterocolitis (NEC) is a gastrointestinal emergency occurring in approximately $1-3 \%$ of neonates admitted to intensive care units. It carries significant mortality (up to $50 \%$ ) and an extensive range of short and long term complications.

Despite decades of research, its pathogenesis remains poorly understood, though current understanding suggests a multi-factorial aetiology. Indeed, prematurity, feeding practises, genetics, various maternal factors and certain neonatal morbidities have all been implicated in the pathogenesis of NEC. As there is a paucity of published surveys providing a general overview of NEC in the last decade, we aimed to provide a more current perspective in a tertiary neonatal unit.

Methods Using diagnostic criteria outlined in the 11th BPSU Annual Report, 49 infants with NEC within a 13 month period were retrospectively identified. Subsequently, data from patient records (including maternal data) and imaging reports was extracted and analysed. The presence of various risk factors (including those mentioned above) and information regarding the presentation, diagnosis and management of NEC in these infants were recorded along with complication and mortality rates.

Results Risk factors including intrauterine growth restriction (18.4\%), patent ductus arteriosus (51\%) and gastroschisis $(10.2 \%)$ were highly prevalent within our cohort. Consistent with previous literature, $86 \%$ of infants were premature and $71.4 \%$ were very low birthweight infants $(<1500 \mathrm{~g})$. However, $24.5 \%$ of infants were from multiple pregnancies which was higher than expected.

Intriguingly, whilst the majority of infants $(75.5 \%)$ were exclusively fed with breast milk initially, by the time NEC was diagnosed this proportion had reduced dramatically $(32.7 \%)$.

Gaseous distension was the most commonly seen radiographic feature (87.8\%) whilst $36.7 \%$ received surgical interventions with nearly all undergoing laparotomy. Gut-related complications were observed in $20.4 \%$ of patients at discharge.

Conclusion This survey provides a more current insight into the progression of NEC from predisposing factors to current management and complications. In agreement with previous studies, no risk factor was uniformly observed. Of particular interest, however, is the trend regarding breast milk feeding. As breast milk has been shown to play a protective role in NEC, it is therefore imperative that support is offered to mothers to encourage continuation of breast milk feeding.

\section{G203(P) TRANSIENT TEMPERATURE GEL ELECTROPHORESIS OF STOOL SAMPLES OF PRETERM INFANTS IN A MULTICENTRE OBSERVATIONAL STUDY}

doi:10.1136/archdischild-2013-304107.215

${ }^{2} \mathrm{~K}$ Brunner, ${ }^{1} \mathrm{LM}$ Beattie, ${ }^{2} \mathrm{~K}$ Gerasimidis, ${ }^{2} \mathrm{DJ}$ Morrison, ${ }^{2} \mathrm{CA}$ Edwards. ${ }^{1}$ Neonatal Unit, Royal Hospital for Sick Children, Yorkhill, UK; ${ }^{2}$ Department of Child Health, University of Glasgow, UK

Aims Despite the effect of enteral feeding on the development of intestinal microbiota in preterm infants remaining poorly understood, trials aiming to prevent necrotising enterocolitis (NEC) using probiotics are well-established. Exclusively breast milk fed preterm infants have a reduced risk of developing NEC and this may be linked with a more 'beneficial' gut microbiota.

Methods The NAPI Study (see abstract BEAT82431) sequentially recruited infants $<32$ weeks and $<1.5 \mathrm{Kg}$ birth weight. Non-meconium faecal samples from the first and fourth weeks of life in 22 infants, 12 with NEC, were analysed by PCR-Transient Temperature Gel Electrophoresis using universal bacterial primers. Species richness and similarities were compared between infants according to feed type: EBM, expressed breast milk, vs Mixed, breast and formula milks.

Results There was large variability between number (1-17) and species diversity (25-36 different species). Number of predominant bacterial species did not increase between the 1st and 4th week of life. Bacterial composition varied largely between the 2 sample points, No difference in species richness or similarity within the 2 feeding groups was observed. 4 bands were identified in $>50 \%$ of infants. Intra-individual similarity varied greatly and ranged from a similarity index (Cs) of $0 \%$ to $66.8 \%$. There was no statistical difference between the similarity indices of the feeding groups $(p=0.8852)$ or between those with and without NEC ( $p=0.1719)$. Conclusion Microbial community of preterm neonates undergoes several interindividual changes during their first month of life. The feeding mode did not seem to have a major impact on the development of bacterial diversity.

\section{G204(P) CAN TAUROLIDINE-BASED CATHETER LOCKS REDUCE CENTRAL VENOUS CATHETER RELATED BLOOD STREAM INFECTIONS IN CHILDREN ON LONG-TERM HOME PARENTERAL NUTRITION?}

doi:10.1136/archdischild-2013-304107.216

L Howarth, E Gaynor, A Rodrigues, PB Sullivan. Department of Paediatric Gastroenterology, Oxford University Hospitals NHS, Oxford, UK

Objectives and study To compare the incidence and characterise the type of catheter-related blood stream infections (CRBSIs) in children with intestinal failure on long-term home parenteral nutrition (PN), using heparin-saline based catheter locks versus those using taurolidine-based catheter locks. There is growing body of evidence that taurolidine-based catheter locks, which have a broadspectrum antimicrobial and antifungal action, is associated with a decreased incidence of CRBSIs children on home PN.

Methods All children referred to a tertiary paediatric gastroenterology service with temporary or on-going intestinal failure requiring long-term PN or preparation for home PN between 2005-2011 were included. Children were given a single-bag system of PN with each infusion via central venous catheter. Parents were formally trained in aseptic techniques and to instil heparin-saline or taurolidinebased solution into the catheter after completion of each infusion. CRBSIs were defined as a laboratory-confirmed blood stream infection from with a peripheral or central venous sample. Results were excluded if evidence that the source of infection was from a second site. All cultures results were confirmed through the microbiology database and clinical records. Research ethics committee approval was sought, but ethical review was not deemed necessary. 